PROCEEDINGS OF THE

AMERICAN MATHEMATICAL SOCIETY

Volume 135, Number 7, July 2007, Pages 1977-1985

S 0002-9939(07)08690-X

Article electronically published on February 28, 2007

\title{
A NEW EXPLICIT EXPRESSION OF THE CONTOU-CARRÈRE SYMBOL
}

\author{
FERNANDO PABLOS ROMO
}

(Communicated by Wen-Ching Winnie Li)

\begin{abstract}
The aim of this note is to offer a new explicit expression of the Contou-Carrère symbol that depends only on a product of a finite number of terms. As an application, we obtain an explicit formula for a Witt Residue.
\end{abstract}

\section{INTRODUCTION}

In 1994 C. Contou-Carrère [4] defined a natural transformation greatly generalizing the tame symbol. In the case of an artinian local base ring $A$ with maximal ideal $m$, the natural transformation takes the following form. Let $f, g \in A((t))^{\times}$be given, where $t$ is a variable. (Here and below $R^{\times}$denotes the multiplicative group of a ring $R$ with unit.) It is possible in exactly one way to write

$$
\begin{aligned}
& f=a_{0} \cdot t^{w(f)} \cdot \prod_{i=1}^{\infty}\left(1-a_{i} t^{i}\right) \cdot \prod_{i=1}^{\infty}\left(1-a_{-i} t^{-i}\right), \\
& g=b_{0} \cdot t^{w(g)} \cdot \prod_{i=1}^{\infty}\left(1-b_{i} t^{i}\right) \cdot \prod_{i=1}^{\infty}\left(1-b_{-i} t^{-i}\right),
\end{aligned}
$$

with $w(f), w(g) \in \mathbb{Z}, a_{i}, b_{i} \in A$ for $i>0, a_{0}, b_{0} \in A^{\times}, a_{-i}, b_{-i} \in m$ for $i>0$, and $a_{-i}=b_{-i}=0$ for $i \gg 0$. By definition, the value of the Contou-Carrère symbol is

$$
\langle f, g\rangle_{A}:=(-1)^{w(f) w(g)} \frac{a_{0}^{w(g)} \prod_{i=1}^{\infty} \prod_{j=1}^{\infty}\left(1-a_{i}^{j /(i, j)} b_{-j}^{i /(i, j)}\right)^{(i, j)}}{b_{0}^{w(f)} \prod_{i=1}^{\infty} \prod_{j=1}^{\infty}\left(1-a_{-i}^{j /(i, j)} b_{j}^{i /(i, j)}\right)^{(i, j)}} \in A^{\times} .
$$

The definition makes sense because only finitely many of the terms appearing in the infinite products differ from 1 . The symbol $\langle\cdot, \cdot\rangle_{A}$ is clearly antisymmetric and, although it is not immediately obvious from the definition, also bimultiplicative.

G. W. Anderson and the author [1] have interpreted the Contou-Carrère symbol $\langle f, g\rangle_{A}$ - up to signs - as a commutator of liftings of $f$ and $g$ to a certain central extension of a group containing $A((t))^{\times}$, and they have exploited the commutator interpretation to prove, in the style of Tate [11, a reciprocity law for the ContouCarrère symbol on a nonsingular complete curve defined over an algebraically closed field $k, A$ being an artinian local $k$-algebra.

Received by the editors February 6, 2006 and, in revised form, February 23, 2006 and March $1,2006$.

2000 Mathematics Subject Classification. Primary 19F15, 19C20.

Key words and phrases. Contou-Carrère symbol, explicit expression, artinian local ring, Witt Residue.

This work was partially supported by the DGESYC research contract no. BFM2003-00078 and Castilla y León regional government contract SA071/04.

(C) 2007 American Mathematical Society 1977 
The author has also obtained a similar result for an algebraic curve over a perfect field [10], and A. Beilinson, S. Bloch and H. Esnault [3] have defined the ContouCarrère symbol as the commutator pairing in a Heisenberg super extension. Moreover, recently, this symbol has played an important role in the work of M. Kapranov and E. Vasserot [6], and M. Asakura 2] has shown that the Contou-Carrère symbol coincides with the boundary map $\delta: K_{i+1}(A((t))) \longrightarrow K_{i}(A)$ described by K. Kato in 7 .

In this context, the goal of this paper is to offer a new explicit expression of the Contou-Carrère symbol that depends only on a product of a finite number of terms (Proposition 3.4). As an application, we study a Witt Residue and we also obtain an explicit expression for it.

\section{Preliminaries}

2.1. Contou-Carrère symbol. Using the theory of groupoids, we can construct a central extension of groups

$$
1 \rightarrow A^{\times} \rightarrow \widetilde{A((t))} \times \stackrel{\pi}{\longrightarrow} A((t))^{\times} \rightarrow 1,
$$

and we have a commutator map

$$
\{\cdot, \cdot\}_{A((t))}^{A[[t]]}: A((t))^{\times} \times A((t))^{\times} \longrightarrow A^{\times} .
$$

That is, if $\tau$ and $\sigma$ are two elements of $A((t))^{\times}$and $\widetilde{\tau}, \widetilde{\sigma} \in \widetilde{A((t))} \times$ are elements such that $\pi(\widetilde{\tau})=\tau$ and $\pi(\widetilde{\sigma})=\sigma$, then the commutator map is

$$
\{\tau, \sigma\}_{A((t))}^{A[[t]]}=\widetilde{\tau} \cdot \widetilde{\sigma} \cdot \widetilde{\tau}^{-1} \cdot \widetilde{\sigma}^{-1} \in A^{\times} .
$$

With the notations of the preceding section, the Contou-Carrère symbol [4] is

$$
\langle f, g\rangle_{A}=(-1)^{w(f) w(g)} \cdot\{f, g\}_{A((t))}^{A[[t]]} .
$$

For details about the central extension (2.1) and the commutator $\{\cdot, \cdot\}_{A(t) \text { (t })}^{A[t]]}$ readers are referred to [1].

For arbitrary elements $f, g, h \in A((t))^{\times}$, the following relations hold:

- $\langle f, g \cdot h\rangle_{A}=\langle f, g\rangle_{A} \cdot\langle f, h\rangle_{A}$.

- $\langle g, f\rangle_{A}=\langle f, g\rangle_{A}^{-1}$.

- $\langle f,-f\rangle_{A}=1$.

- Given $\varphi \in A((t))^{\times}$with positive winding number $n$, one has that

$$
\langle f, g \circ \varphi\rangle_{A}=\left\langle\mathcal{N}_{\varphi}[f], g\right\rangle_{A},
$$

where $\mathcal{N}_{\varphi}: A((t))^{\times} \rightarrow A((t))^{\times}$denotes the corresponding norm mapping: viewing $A((t))$ via the homomorphism $h \mapsto h \circ \varphi$ as a free $A((t))$-module of rank $n$ ([9], Proposition 3.6).

Remark 2.1. With the notation of the previous section, we should note that the original expression of the Contou-Carrère symbol [4] is

$$
\langle f, g\rangle_{A}=(-1)^{w(f) w(g)} \frac{a_{0}^{w(g)} \cdot \exp \left(\sum_{i>0}\left[\delta_{i}(f) \cdot \delta_{-i}(g) / i\right]\right)}{b_{0}^{w(f)} \cdot \exp \left(\sum_{i>0}\left[\delta_{-i}(f) \cdot \delta_{i}(g) / i\right]\right)},
$$

where $\delta_{m}(f)=\operatorname{Res}\left(t^{m} \frac{d f}{f}\right)$. 
Let $\xi_{1}, \xi_{2}, \ldots ; \eta_{1}, \eta_{2}, \ldots$ now be indeterminates. Recall from [5] that we can define the sequences $\bar{x}=\left(\bar{x}_{i}\right)$ and $\bar{y}=\left(\bar{y}_{j}\right)$ by the equations

$$
\begin{aligned}
& \prod_{i}\left(1-\xi_{i} t\right)=1+\bar{x}_{1} t+\bar{x}_{2} t^{2}+\ldots, \\
& \prod_{i}\left(1-\eta_{i} t\right)=1+\bar{y}_{1} t+\bar{y}_{2} t^{2}+\ldots
\end{aligned}
$$

Hence, from these definitions we can construct a polynomial sequence $P_{1}, P_{2}, \ldots$ satisfying the relations

$$
\prod_{i, j}\left(1-\xi_{i} \eta_{j} t\right)=1+P_{1} t+P_{2} t^{2}+\ldots
$$

Bearing in mind the fundamental theorem of symmetric functions, $P_{j}$ can be written as $P_{j}\left(\bar{x}_{1}, \ldots, \bar{x}_{j} ; \bar{y}_{1}, \ldots, \bar{y}_{j}\right)$.

Thus, if $A$ is a commutative ring, we can consider on the multiplicative group

$$
\bigwedge(A)=\left\{1+a_{1} t+a_{2} t^{2}+\ldots, \quad a_{i} \in A\right\} \subseteq A[[t]]
$$

a second operation by means of the formula

$$
\left(1+a_{1} t+a_{2} t^{2}+\ldots\right) *\left(1+b_{1} t+b_{2} t^{2}+\ldots\right)=1+P_{1}(a, b) t+P_{2}(a, b) t^{2}+\ldots,
$$

such that $(\bigwedge(A), \cdot, *)$ is a ring.

Moreover, $\mathbb{W}(A)$ being the ring of Witt vectors with coefficients in $A$, we can define a map

$$
\begin{aligned}
& E_{A}: \mathbb{W}(A) \longrightarrow \bigwedge(A), \\
& \left(a_{1}, a_{2}, \ldots\right) \longrightarrow \prod_{i \geq 1}\left(1-a_{i} t^{i}\right),
\end{aligned}
$$

which is an isomorphism of rings because $E_{A}(a+b) E_{A}(a) \cdot E_{A}(b)$ and $E_{A}(a \cdot b)=$ $E_{A}(a) * E_{A}(b)$.

Since $\mathbb{W}(A)$ is a commutative ring and with unit element ([5], page 117), we can denote by $\mathbb{W}_{+}(A)$ the abelian group induced by the ring structure.

Furthermore, we can define the abelian group

$$
\hat{\mathbb{W}}_{+}(A)=\left\{\begin{array}{c}
\left(b_{1}, b_{2}, \ldots\right) \in \mathbb{W}_{+}(A) \text { with } b_{i} \text { nilpotent } \\
\text { for all } i \text { and } b_{i}=0 \text { for almost all } i
\end{array}\right\}
$$

If the artinian local ring $A$ is a $k$-algebra with $\operatorname{ch}(k)=0$, bearing in mind Proposition $2.4([10]$, p. 44$)$ and denoting $E_{A}(\cdot)=E_{A}(\cdot, t)$, we have that

$$
\exp \left(\sum_{i>0}\left[\delta_{i}(f) \cdot \delta_{-i}(g) / i\right]\right)=E_{A}\left(a \cdot b^{\prime}, 1\right),
$$

where $a=\left(a_{1}, a_{2}, \ldots\right) \in \mathbb{W}_{+}(A)$ and $b^{\prime}=\left(b_{-1}, b_{-2}, \ldots\right) \in \hat{\mathbb{W}}_{+}(A)$, with the notation of (1.1).

Remark 2.2. If $A$ is again an artinian local $k$-algebra with $\operatorname{ch}(k)=0$, let us now consider again $f, g \in A((t))^{\times}$and let us assume that there exist decompositions

$$
\begin{aligned}
& f \bar{a}_{0} \cdot t^{w(f)} \cdot \prod_{i \geq 1}\left(1-\bar{a}_{i} t\right) \cdot \prod_{i \geq 1}\left(1-\bar{a}_{-i} t^{-1}\right), \\
& g=\bar{b}_{0} \cdot t^{w(g)} \cdot \prod_{j \geq 1}\left(1-\bar{b}_{j} t\right) \cdot \prod_{j \geq 1}\left(1-\bar{b}_{-j} t^{-1}\right),
\end{aligned}
$$

with $\bar{a}_{i}, \bar{b}_{j} \in A$ and $\bar{a}_{-i}, \bar{b}_{-j} \in m$. 
It follows from the above considerations, in particular from equality (2.4), and from the results of [8] (formulas (4.1) on p. 62 and (4.3) on p. 63), that

$$
\begin{aligned}
& \exp \left(\sum_{i>0}\left[\delta_{i}(f) \cdot \delta_{-i}(g) / i\right]\right)=\left[\sum_{\lambda} z_{\lambda}^{-1} p_{\lambda}(\bar{a}) p_{\lambda}\left(\bar{b}^{\prime}\right)\right]^{-1}, \\
& \exp \left(\sum_{i>0}\left[\delta_{i}(f) \cdot \delta_{-i}(g) / i\right]\right)=\left[\sum_{\lambda} s_{\lambda}(\bar{a}) s_{\lambda}\left(\bar{b}^{\prime}\right)\right]^{-1},
\end{aligned}
$$

summed over all partitions $\lambda=\left(\lambda_{1}, \lambda_{2}, \ldots\right)$, with:

- $\bar{a}=\left(\bar{a}_{1}, \bar{a}_{2}, \ldots\right)$;

- $\bar{b}^{\prime}=\left(\bar{b}_{-1}, \bar{b}_{-2}, \ldots\right)$;

- $z_{\lambda}=\prod_{i \geq 1} i^{m_{i}} \cdot m_{i}$ !, where $m_{i}=m_{i}(\lambda)$ is the number of parts of $\lambda$ equal to $i$;

- $p_{\lambda}=p_{\lambda_{1}} \cdot p_{\lambda_{2}} \ldots$, where $p_{\lambda_{i}}(x)$ is the $\lambda_{i}$-th power sum $\sum x_{j}^{\lambda_{i}}$;

- $s_{\lambda}$ is the Schur function associated with the partition $\lambda$.

Therefore, setting $\bar{b}=\left(\bar{b}_{1}, \bar{b}_{2}, \ldots\right)$ and $\bar{a}^{\prime}=\left(\bar{a}_{-1}, \bar{a}_{-2}, \ldots\right)$, equivalent expressions to (2.3) are:

(1) $\langle f, g\rangle_{A}=(-1)^{w(f) w(g)} \frac{\bar{a}_{0}^{w(g)} \cdot \sum_{\lambda} z_{\lambda}^{-1} p_{\lambda}(\bar{b}) p_{\lambda}\left(\bar{a}^{\prime}\right)}{\bar{b}_{0}^{w(f)} \cdot \sum_{\lambda} z_{\lambda}^{-1} p_{\lambda}(\bar{a}) p_{\lambda}\left(\bar{b}^{\prime}\right)}$

(2) $\langle f, g\rangle_{A}(-1)^{w(f) w(g)} \frac{\bar{a}_{0}^{w(g)} \cdot \sum_{\lambda} s_{\lambda}(\bar{b}) s_{\lambda}\left(\bar{a}^{\prime}\right)}{\bar{b}_{0}^{w(f)} \cdot \sum_{\lambda} s_{\lambda}(\bar{a}) s_{\lambda}\left(\bar{b}^{\prime}\right)}$.

2.2. Witt Residue symbol. Similar to [1, let

$$
\{\epsilon\} \coprod\left\{\mathbf{x}_{i}, \mathbf{y}_{i}\right\}_{i=1}^{\infty}
$$

be a family of independent variables. Let us write

$$
\begin{gathered}
\prod_{i=1}^{\infty}\left(\left(1-\mathbf{x}_{i} \epsilon^{i}\right)\left(1-\mathbf{y}_{i} \epsilon^{i}\right)\right)=\prod_{i=1}^{\infty}\left(1-\mathbf{A}_{i} \epsilon^{i}\right), \\
\prod_{i=1}^{\infty} \prod_{j=1}^{\infty}\left(1-\mathbf{x}_{i}^{j /(i, j)} \mathbf{y}_{j}^{i /(i, j)} \epsilon^{i j /(i, j)}\right)^{(i, j)} \prod_{i=1}^{\infty}\left(1-\mathbf{M}_{i} \epsilon^{i}\right),
\end{gathered}
$$

thereby defining families of polynomials

$$
\left\{\mathbf{A}_{n}, \mathbf{M}_{n} \in \mathbb{Z}\left[\left\{\mathbf{x}_{i}, \mathbf{y}_{i}\right\}_{i \mid n}\right]\right\}_{n=1}^{\infty} .
$$

For any commutative ring $B$ with unit and finite subset $\Delta$ of the set of positive integers closed under passage to divisors, let $\mathbb{W}_{\Delta}(B)$ denote the set of vectors with entries in $B$ indexed by $\Delta$. It can be shown that the $\mathbf{A}$ 's and $\mathbf{M}$ 's define addition and multiplication laws with respect to which $\mathbb{W}_{\Delta}(B)$ becomes a commutative ring with unit, functorially in commutative rings $B$ with unit.

Let us fix a positive integer $N$. If $\mathbb{W}_{\leq N}(B)$ is the ring of Witt vectors associated with $\Delta=\{1,2 \ldots, N\}$, one has that the map

$$
x=\left(x_{i}\right)_{i=1}^{N} \mapsto \prod_{i=1}^{N}\left(1-x_{i} \epsilon^{i}\right) \bmod \epsilon^{N+1}
$$

identifies the additive group underlying $\mathbb{W}_{\leq N}(B)$ with the group of units in the ring $B[\epsilon] /\left(\epsilon^{N+1}\right)$ congruent to 1 modulo $(\epsilon)$ functorially in commutative rings $B$ with unit.

If $F$ is a field, and $A:=F[\epsilon] /\left(\epsilon^{N+1}\right)$, we can define a pairing

$$
\operatorname{Res}_{\leq N}^{\mathbb{W}}(\cdot, \cdot): F((t))^{\times} \times \mathbb{W}_{\leq N}(F((t))) \rightarrow \mathbb{W}_{\leq N}(F)
$$


by the rule

$$
\left\langle f, \prod_{i=1}^{N}\left(1-x_{i} \epsilon^{i}\right)\right\rangle_{A((t))^{\times}} \equiv \prod_{i=1}^{N}\left(1-\epsilon^{i}\left[\operatorname{Res}_{\leq N}^{\mathbb{W}}(f, x)\right]_{i}\right) \bmod \left(\epsilon^{N+1}\right),
$$

where $\langle\cdot, \cdot\rangle_{A((t)) \times}$ is the Contou-Carrère symbol.

The pairing Res $\mathbb{W}_{\leq N}^{\mathbb{W}}$ is essentially the pairing introduced in Witt's paper [12].

Hence, when $N=1$ we have a map

$$
\operatorname{Res}^{\mathbb{W}}(\cdot, \cdot): F((t))^{\times} \times F((t)) \rightarrow F,
$$

where

$$
\langle f, 1-\epsilon g\rangle_{A((t)) \times} \equiv 1-\epsilon\left[\operatorname{Res}^{\mathbb{W}}(f, g)\right] \bmod \left(\epsilon^{2}\right),
$$

for all $f \in F((t))^{\times}$and $g \in F((t))$.

One has that:

- $\operatorname{Res}^{\mathbb{W}}\left(f \cdot f^{\prime}, g\right)=\operatorname{Res}^{\mathbb{W}}(f, g)+\operatorname{Res}^{\mathbb{W}}\left(f^{\prime}, g\right)$.

- $\operatorname{Res}^{\mathbb{W}}\left(f, g+g^{\prime}\right)=\operatorname{Res}^{\mathbb{W}}(f, g)+\operatorname{Res}^{\mathbb{W}}\left(f, g^{\prime}\right)$.

3. New explicit expression of the Contou-Carrère Symbol

Given an element $f \in A((t))^{\times}$, it is possible in exactly one way to write

$$
f=a_{0} \cdot t^{w(f)} \cdot \prod_{i=1}^{\infty}\left(1-a_{-i} t^{-i}\right) \cdot \prod_{i=1}^{\infty}\left(1-a_{i} t^{i}\right)
$$

where

$$
w(f) \in \mathbb{Z}, \begin{cases}a_{i}=0 & \text { if } i \ll 0, \\ a_{i} \in m & \text { if } i<0, \\ a_{i} \in A^{\times} & \text {if } i=0, \\ a_{i} \in A & \text { if } i>0 .\end{cases}
$$

The integer number $w(f)$ is the winding number of $f$, and the set $\left\{a_{i}\right\}_{i=-\infty}^{\infty}$ is the family of Witt parameters of $f$.

Thus, we can consider the morphism of groups

$$
\begin{aligned}
\phi: A((t))^{\times} & \longrightarrow A((t))^{\times}, \\
f & \longmapsto a_{0} .
\end{aligned}
$$

Remark 3.1 (Characterization of the Contou-Carrère symbol). It follows from the properties described in Subsection 2.1 that the commutator map $\{\cdot, \cdot\}_{A((t))}^{A[t]]}$ is the only bimultiplicative map

$$
\{\cdot, \cdot\}_{A((t))}^{A[[t]]}: A((t))^{\times} \times A((t))^{\times} \longrightarrow A^{\times}
$$

that satisfies the conditions:

- $\{\cdot, \cdot\}_{\left.A((t))\right|_{A[[t]] \times \times A[t]] \times} ^{A[t]]}}=1$.

- $\{f, g\}_{A((t))}^{A[[t]]}=\phi\left(\mathcal{N}_{g}[f]\right)$ for all $g \in A((t))^{\times}$with $w(g)>0$.

Hence, the Contou-Carrère symbol is the only map

$$
\langle\cdot, \cdot\rangle_{A}: A((t))^{\times} \times A((t))^{\times} \longrightarrow A^{\times}
$$

that satisfies the properties:

- $\langle\cdot, \cdot\rangle_{A}$ is bimultiplicative.

- $\langle\cdot, \cdot\rangle_{A_{\left.\left.\right|_{A[t]]} \times \times A[t]\right] \times}}=1$. 
- $\langle f, g\rangle_{A}=(-1)^{w(f) w(g)} \cdot \phi\left(\mathcal{N}_{g}[f]\right)$ for all $g \in A((t))^{\times}$with $w(g)>0$.

As far as we know a characterization relating the Contou-Carrere symbol with a norm map is not stated explicitly in the literature.

Let us now consider a series $s(t) \in A[[t]]^{\times}$and an element $a \in m$. For each positive integer $n$, setting

$$
s(t)=s_{0}^{n}\left(t^{n}\right)+t \cdot s_{1}^{n}\left(t^{n}\right)+\cdots+t^{n-1} \cdot s_{n-1}^{n}\left(t^{n}\right),
$$

we can construct a matrix $C_{s(t)}^{n, a} \in \operatorname{Gl}(n, A)$ where the coefficients are

$$
\left[C_{s(t)}^{n, a}\right]_{i j}=\left\{\begin{array}{r}
s_{i-j}^{n}(a) \text { if } i \geq j, \\
a \cdot s_{j-i}^{n}(a) \text { if } i<j .
\end{array}\right.
$$

That is, the expression of the matrix is

$$
C_{s(t)}^{n, a}\left(\begin{array}{ccccc}
s_{0}^{n}(a) & a \cdot s_{n-1}^{n}(a) & \ldots & a \cdot s_{2}^{n}(a) & a \cdot s_{1}^{n}(a) \\
s_{1}^{n}(a) & s_{0}^{n}(a) & \ldots & a \cdot s_{3}^{n}(a) & a \cdot s_{2}^{n}(a) \\
\vdots & \ddots & \ddots & \vdots & \vdots \\
s_{n-2}^{n}(a) & \ldots & s_{1}^{n}(a) & s_{0}^{n}(a) & a \cdot s_{n-1}^{n}(a) \\
s_{n-1}^{n}(a) & \ldots & s_{2}^{n}(a) & s_{1}^{n}(a) & s_{0}^{n}(a)
\end{array}\right) .
$$

Note that in (3.1) the series $s_{0}^{n}(t) \in A[[t]]^{\times}$. Moreover, for all $s(t) \in A[[t]]^{\times}$and $a \in m$, one has that

$$
\operatorname{det} C_{s(t)}^{1, a}=\phi(s(t))=s(a) .
$$

We shall now give an explicit expression of the Contou-Carrere symbol by using the determinants of the matrices $C_{s(t)}^{n, a}$.

Lemma 3.2. If $n>0, a \in m$ and $s(t) \in A[[t]]^{\times}$, one has that

$$
\left\langle s(t), t^{n}-a\right\rangle_{A}=\operatorname{det} C_{s(t)}^{n, a} .
$$

Proof. Viewing $A((t))$ via the homomorphism $h \mapsto h \circ\left(t^{n}-a\right)$ as a free $A((t))$ module of rank $n$ with basis $\left\{1, \ldots, t^{n-1}\right\}$, from the relations

$$
\begin{gathered}
s_{i}^{n}\left(t^{n}\right)=s_{i}^{n}(a)+\left(t^{n}-a\right) \cdot \widetilde{s}_{i}^{n}\left(t^{n}-a\right), \\
t^{n} \cdot s_{j}^{n}\left(t^{n}\right)=a \cdot s_{j}^{n}(a)+\left(t^{n}-a\right) \cdot \widetilde{s}_{j}^{n}\left(t^{n}-a\right),
\end{gathered}
$$

we have that the matrix of the homothety $h_{s(t)}$ is obtained from the equality

$$
\begin{aligned}
& h_{s(t)}(1)=s_{0}^{n}\left(t^{n}\right)+t \cdot s_{1}^{n}\left(t^{n}\right)+\cdots+t^{n-1} \cdot s_{n-1}^{n}\left(t^{n}\right) \\
& =\left[\left[s_{0}^{n}(a)+t \cdot \widetilde{s}_{0}^{n}(t)\right] \circ\left(t^{n}-a\right)\right]+\cdots+t^{n-1} \cdot\left[\left[s_{n-1}^{n}(a)+t \cdot \widetilde{s}_{n-1}^{n}(t)\right] \circ\left(t^{n}-a\right)\right] \\
& \equiv\left(s_{0}^{n}(a)+t \cdot \widetilde{s}_{0}^{n}(t), \ldots, s_{n-1}^{n}(a)+t \cdot \widetilde{s}_{n-1}^{n}(t)\right)
\end{aligned}
$$

and from the expressions

$$
\begin{aligned}
& h_{s(t)}\left(t^{i}\right)=t^{i} \cdot s_{0}^{n}\left(t^{n}\right)+\cdots+t^{n-1} \\
& \quad \cdot s_{n-1-i}^{n}\left(t^{n}\right)+t^{n} \cdot s_{n-i}^{n}\left(t^{n}\right)+\cdots+t^{i-1} \cdot t^{n} \cdot s_{n-1}^{n}\left(t^{n}\right) \\
& =t^{i} \cdot\left[\left[s_{0}^{n}(a)+t \cdot \widetilde{s}_{0}^{n}(t)\right] \circ\left(t^{n}-a\right)\right]+\cdots+t^{i-1} \\
& \cdot \cdot\left[\left[a \cdot s_{n-1}^{n}(a)+t \cdot \widetilde{s}_{n-1}^{n}(t)\right] \circ\left(t^{n}-a\right)\right] \\
& \equiv\left(a \cdot s_{n-i}^{n}(a)+t \cdot \widetilde{s}_{n-i}^{n}(t), \ldots, s_{n-1-i}^{n}(a)+t \cdot \widetilde{s}_{n-1-i}^{n}(t)\right)
\end{aligned}
$$

when $i \geq 1$. 
Thus, bearing in mind the definition of $C_{s(t)}^{n, a}$, since $\mathcal{N}_{t^{n}-a}[s(t)] \in A[[t]]^{\times}$and

$$
\left\langle s(t), t^{n}-a\right\rangle_{A}=\phi\left(\mathcal{N}_{t^{n}-a}[s(t)]\right),
$$

the claim is deduced.

A direct consequence of Lemma 3.2 is the following result that appeared in [2].

Corollary 3.3. For every $s(t) \in A[[t]]^{\times}$and $a \in m$, one has that

$$
\langle s(t), t-a\rangle_{A}=s(a) .
$$

Given two elements $f, g \in A((t))^{\times}$, let us now write

$$
f=t^{-N} \cdot s(t) \cdot \prod_{i=1}^{k}\left(t^{i}-a_{-i}\right), \quad g=t^{-M} \cdot s^{\prime}(t) \cdot \prod_{j=1}^{h}\left(t^{j}-b_{-j}\right),
$$

with $N, M \in \mathbb{Z}^{+} ; a_{-i}, b_{-j} \in m ; s(t), s^{\prime}(t) \in A[[t]]^{\times} ; w(f)=\frac{k \cdot(k+1)}{2}-N$ and $w(g)=\frac{h \cdot(h+1)}{2}-M$.

Proposition 3.4 (Explicit expression of the Contou-Carrère symbol). With the previous notation, if $\phi(f)=a_{0}$ and $\phi(g)=b_{0}$, the Contou-Carrère symbol is

$$
\langle f, g\rangle_{A}=(-1)^{w(f) w(g)} \cdot \frac{b_{0}^{M} \cdot \prod_{j=1}^{h} \operatorname{det} C_{s(t)}^{j, b_{-j}}}{a_{0}^{N} \cdot \prod_{i=1}^{k} \operatorname{det} C_{s^{\prime}(t)}^{i, a_{-i}}} .
$$

Proof. The expression follows from Lemma 3.2, bearing in mind that

- $\left\langle t^{-N}, t^{-M}\right\rangle_{A}=(-1)^{N \cdot M}$;

- $\left\langle t^{-N}, t^{j}-b_{-j}\right\rangle_{A}=(-1)^{j \cdot N}$;

- $\left\langle t^{i}-a_{-i}, t^{-M}\right\rangle_{A}=(-1)^{i \cdot M}$;

- $\left\langle t^{-N}, s^{\prime}(t)\right\rangle_{A}=b_{0}^{-M}$;

- $\left\langle s(t), t^{-M}\right\rangle_{A}=a_{0}^{-N}$;

- $\left\langle t^{i}-a_{-i}, t^{j}-b_{-j}\right\rangle_{A}=(-1)^{i \cdot j}$.

Corollary 3.5. Given two elements $f, g \in A((t))^{\times}$, such that

$$
f=t^{-N} \cdot a_{0} \cdot \bar{s}(t) \cdot \prod_{i=1}^{k}\left(t^{i}-a_{-i}\right), \quad g=t^{-M} \cdot b_{0} \cdot \bar{s}^{\prime}(t) \cdot \prod_{j=1}^{h}\left(t^{j}-b_{-j}\right),
$$

with $N, M \in \mathbb{Z}^{+} ; a_{0}, b_{0} \in A^{\times} ; a_{-i}, b_{-j} \in m ; \bar{s}(t), \bar{s}^{\prime}(t) \in A[[t]]^{\times} ; w(f)=\frac{k \cdot(k+1)}{2}-$ $N$ and $w(g)=\frac{h \cdot(h+1)}{2}-M$, the Contou-Carrère symbol is

$$
\langle f, g\rangle_{A}=(-1)^{w(f) w(g)} \cdot \frac{a_{0}^{w(g)} \cdot \prod_{j=1}^{h} \operatorname{det} C_{\bar{s}(t)}^{j, b_{-j}}}{b_{0}^{w(f)} \cdot \prod_{i=1}^{k} \operatorname{det} C_{\bar{s}^{\prime}(t)}^{i, a_{-i}}} .
$$

Proof. Since $\left\langle a_{0}, t^{j}-b_{-j}\right\rangle_{A}=a_{0}^{j}$ and $\left\langle t^{i}-a_{-i}, b_{0}\right\rangle_{A}=b_{0}^{i}$, this formula follows immediately from the explicit expression given in Proposition 3.4

Finally, as an application of this explicit formula of the Contou-Carrère symbol, we shall study the map $\operatorname{Res}^{\mathbb{W}}(\cdot, \cdot): F((t))^{\times} \times F((t)) \rightarrow F$ defined in Subsection 2.2 by the rule

$$
\langle f, 1-\epsilon g\rangle_{A((t)) \times} \equiv 1-\epsilon\left[\operatorname{Res}^{\mathbb{W}}(f, g)\right] \bmod \left(\epsilon^{2}\right) .
$$


Note that if $g=\sum_{j \geq-h} b_{j} t^{j}$, then

$$
1-\epsilon g=t^{-\frac{h(h+1)}{2}} \cdot\left(1-\epsilon b_{0}\right) \cdot \bar{s}^{\prime}(t) \cdot \prod_{j=1}^{h}\left(t^{j}-\epsilon b_{-j}\right),
$$

where $\bar{s}^{\prime}(t) \in A[[t]]^{\times}$with $A=F[\epsilon] /\left(\epsilon^{2}\right)$.

Thus, if $f=t^{w(f)} \cdot a_{0} \cdot \bar{s}(t)$, we have that

$$
\langle f, 1-\epsilon g\rangle_{A((t)) \times}=\frac{\prod_{j=1}^{h} \operatorname{det} C_{\bar{s}(t)}^{j, \epsilon b_{-j}}}{\left[1-\epsilon b_{0}\right]^{w(f)}}=\frac{\prod_{j=1}^{h} \operatorname{det} C_{\bar{s}(t)}^{j, \epsilon b_{-j}}}{\left[1-\epsilon w(f) b_{0}\right]} .
$$

Accordingly, if $\operatorname{Tr} A$ denotes the trace of a square matrix $A$, bearing in mind that

$$
C_{\bar{s}(t)}^{j, \epsilon b_{-j}}=A_{\bar{s}(t)}^{j} \cdot\left(\operatorname{Id}+\epsilon \cdot b_{-j} \cdot\left[A_{\bar{s}(t)}^{j}\right]^{-1} \cdot B_{\bar{s}(t)}^{j}\right),
$$

with

and

$$
A_{\bar{s}(t)}^{j}=\left(\begin{array}{ccccc}
1 & 0 & \ldots & \ldots & 0 \\
a_{1} & 1 & 0 & \ldots & 0 \\
\vdots & \ddots & \ddots & \ldots & \vdots \\
a_{j-2} & \ldots & a_{1} & 1 & 0 \\
a_{j-1} & \ldots & a_{2} & a_{1} & 1
\end{array}\right)
$$

$$
B_{\bar{s}(t)}^{j}=\left(\begin{array}{ccccc}
a_{j} & a_{j-1} & \ldots & \ldots & a_{1} \\
a_{j+1} & a_{j} & a_{j-1} & \ldots & a_{2} \\
\vdots & \ddots & \ddots & \ldots & \vdots \\
a_{2 j-2} & \ldots & a_{j+1} & a_{j} & a_{j-1} \\
a_{2 j-1} & \cdots & a_{j+2} & a_{j+1} & a_{j}
\end{array}\right),
$$

where $\bar{s}(t)=1+\sum_{i \geq 1} a_{i} \cdot t^{i}$, we have that

$$
\operatorname{det} C_{\bar{s}(t)}^{j, \epsilon b_{-j}}=1+\epsilon \cdot b_{-j} \cdot \operatorname{Tr}\left(\left[A_{\bar{s}(t)}^{j}\right]^{-1} \cdot B_{\bar{s}(t)}^{j}\right) .
$$

Hence, since

$$
\left(\left[A_{\bar{s}(t)}^{j}\right]^{-1}\right)_{\alpha \beta}=\left\{\begin{array}{cc}
1 & \text { if } \alpha=\beta, \\
0 & \text { if } \alpha<\beta, \\
\sum_{\substack{0 \\
i_{1}+\cdots+i_{t}=\alpha-\beta \\
1 \leq i_{1}, \ldots, i_{t} \leq j-1}}(-1)^{t} a_{i_{1}} \cdots a_{i_{t}} & \text { if } \alpha>\beta,
\end{array}\right.
$$

then

$$
\operatorname{Tr}\left(\left[A_{\bar{s}(t)}^{j}\right]^{-1} \cdot B_{\bar{s}(t)}^{j}\right)=j \cdot a_{j}+\sum_{r=1}^{j-1}\left[\sum_{\substack{i_{1}+\cdots+i_{t}=r \\ 1 \leq i_{1}, \ldots, i_{t} \leq j-1}}(-1)^{t} a_{i_{1}} \cdots a_{i_{t}}\right] a_{j-r},
$$

and the explicit expression of the Witt Residue is:

$$
\operatorname{Res}^{\mathbb{W}}(f, g)=-w(f) \cdot b_{0}-\sum_{j=1}^{h} b_{-j} \cdot\left(j \cdot a_{j}+\sum_{r=1}^{j-1}\left[\sum_{\substack{i_{1}+\cdots+i_{t}=r \\ 1 \leq i_{1}, \ldots, i_{t} \leq j-1}}(-1)^{t} a_{i_{1}} \cdots a_{i_{t}}\right] a_{j-r}\right)
$$

for $f=t^{w(f)} \cdot a_{0} \cdot\left[1+\sum_{i \geq 1} a_{i} \cdot t^{i}\right]$ and $g=\sum_{j \geq-h} b_{j} t^{j}$. 
Remark 3.6 (Residue Theorem). Let $X$ be a nonsingular and irreducible curve over the field $F$ and let $\Sigma_{X}$ be its function field. Fixing a closed point $x \in X$, we have an immersion of rings

$$
i_{x}: \Sigma_{X} \hookrightarrow\left(\widehat{\mathcal{O}_{x}}\right)_{0} \simeq F((t)),
$$

such that, by restriction of $\operatorname{Res}^{\mathbb{W}}(\cdot, \cdot)$, we can consider the map

$$
\operatorname{Res}_{x}^{\mathbb{W}}(\cdot, \cdot): \Sigma_{X}^{\times} \times \Sigma_{X} \rightarrow F
$$

which is the Witt Residue associated with the closed point $x$.

When $X$ is a complete curve, a direct consequence of the reciprocity law for the Contou-Carrère symbol [1] is the following Residue Theorem:

$$
\sum_{x \in X} \operatorname{Res}_{x}^{\mathbb{W}}(f, g)=0
$$

for every $f \in \Sigma_{X}^{\times}$and $g \in \Sigma_{X}$.

\section{ACKNOWLEDGMENT}

The author thanks the anonymous referee for her/his constructive comments aimed at improving the preliminary version of this paper.

\section{REFERENCES}

[1] Anderson, G. W.; Pablos Romo, F. Simple Proofs of Classical Explicit Reciprocity Laws on Curves using Determinant Grupoids over an Artinian Local Ring. Comm. Algebra. 2004, 32(1), 79-102. MR2036223 (2005d:11099)

[2] Asakura, M. On dlog image of $K_{2}$ of elliptic surface minus singular fibers. Preprint, 2005, math. AG./0511190.

[3] Beilinson, A.; Bloch S.; Esnault H. $\epsilon$-factors for Gauss-Manin determinants. Moscow Math. J. 2002, 2(3), 477-532. MR1988970 (2004m:14011)

[4] Contou-Carrere, C. Jacobienne Locale, Groupe de Bivecteurs de Witt Universel et Symbole Modéré. C.R. Acad. Sci. Paris. 1994, 318, Série I, 743-746. MR1272340 (95c:14059)

[5] Hazewinkel, M., Formal Groups and Applications, Academic Press, New York, San Francisco, London, 1978. MR0506881 (82a:14020)

[6] Kapranov, M.; Vasserot, E. Formal loops groups II: A local Riemann-Roch Theorem for determinantal gerbes. Preprint, 2005, math. AG./0509646.

[7] Kato, K. A Generalization of local class field theory by using $K$-groups II. J. Fac. Sci. Univ. Tokyo Sect. IA 1980, 27(3), 603-683. MR0603953 (83g:12020a)

[8] Macdonald, I. G., Symmetric Functions and Hall Polynomials, Oxford Science Publications, second edition, Oxford, 1995. MR1354144 (96h:05207)

[9] Pablos Romo, F. A Contou-Carrère symbol on $\mathrm{Gl}(n, A((t)))$ and a Witt Residue Theorem on $\operatorname{Mat}\left(n, \Sigma_{C}\right)$. Int. Math. Res. Not. 2006, Article ID 56824, 1-21. MR2211145 (2006m:11092)

[10] Pablos Romo, F. A Generalization of the Contou-Carrère symbol. Israel J. Math. 2004, 141, 39-60. MR2063024 (2005g:11115)

[11] Tate, J. Residues of Differentials on Curves. Ann. Scient. Éc. Norm. Sup. 1968, 4a série, 1, 149-159. MR0227171 (37:2756)

[12] Witt, E. Zyklische Körper und Algebren der Charakteristik $p$ vom Grad $p^{n}$. J. Reine Angew. Math. 1937, 176, 126-140.

Departamento de Matemáticas, Universidad de Salamanca, Plaza de la Merced 1-4, 37008 Salamanca, España

E-mail address: fpablos@usal.es 